\title{
Psychosocial impact of the lockdown and COVID-19
}

\author{
Avinash De Sousa ${ }^{1 *}$, Sai Krishna $\mathbf{P}^{2}$ \\ ${ }^{1}$ Consultant Psychiatrist and Research Associate, Dept. of Psychiatry, Lokmanya Tilak Municipal Medical College, Mumbai, \\ Maharashtra, ${ }^{2}$ Professor, Dept. of Psychiatry, PIMS, Karimnagar, Telangana, India \\ *Corresponding Author: Avinash De Sousa \\ Email: avinashdes888@gmail.com
}

\section{Introduction}

The COVID-19 pandemic resulted in a nationwide lockdown initially for 21 days and was extended to control further spread of the deadly disease. National and international travel was affected and people were ordered to stay indoors while moving out sparingly for essentials only. The lockdown is a unique phenomenon on its own. It is for the first time in recent years in our nation that we are homebound with our family members. This is very different from quarantine where the patient is isolated at home or in a quarantine facility and does not get to meet his family members. The lockdown while does grant us some freedom, has huge psychological ramifications as well for the common man as well as for patients who already suffer or are predisposed to psychopathology.

\section{Psychosocial factors in a Lockdown}

There are a number of psychosocial factors that shall determine one's response to a lockdown. The lockdown would reduce income of most professions and bring financial stress on anyone if it would continue for a long time. Sufferers are mostly the daily wage earners and those that have businesses and establishments that earn on a daily basis. The longer the lockdown, the greater the financial strain and this would lead to depression, anxiety, uncertainty about the future and days ahead which would cause panic and further anxiousness. The person with financial liabilities would succumb more to the same during the lockdown period and this would lead increased severity of depressive symptoms and an increased frequency of panic attacks. ${ }^{1}$

\section{Corona-anxiety}

Many people developed what is called "Corona anxiety" i.e., they feel that they may contract the virus. Symptoms like mild fever and cough may be thought of as COVID and people started experiencing anxiety symptoms. The thought of stepping out may precipitate anxiety and this will be more in people with personalities that are predisposed to anxiety and worry. In those who may get infected the knowledge of the diagnosis, and isolation for treatment can be a potential trigger for anxiety and depression. Excessive protection and obsessiveness about social distancing and protection may also develop in people. ${ }^{2}$

\section{Psychological Aspects of Undergoing Quarantine}

Most reviewed studies on psychological effects of quarantine have reported negative psychological effects including post-traumatic stress symptoms, confusion, anxiety, depression and anger. The stress revolved around factors like longer quarantine duration, staying away from family, infection fear, boredom, inadequate supplies, inadequate information, financial loss, isolation and stigma. Patients need to be psycho-educated about the clear rationale for quarantine and information about protocols, and ensure sufficient supplies are provided. If educated properly, people can appreciate the benefits of quarantine to wider society. Considering the potential risk of agitation in an isolated environment, it is advisable to allow the patient to interact with the family member/caregiver using video/audio call whenever a patient is under quarantine or admitted to COVID hospital. ${ }^{3-4}$

\section{Access to Proper Mental Health Care}

The biggest problem during COVID-19 would be access to proper mental health care. Patients would not be able to reach a mental health professional and a majority of consultations would happen over the phone and in India, telepsychiatry as a modality is yet to kick off in a big way. The psychiatric examination and mental status examination are best done in person and a teleconference video call may not suffice for the same. Many agencies have started telepsychiatry services offering free mental health services during the COVID-19 lockdown. Thus, while services are available, there is no stringent body that would monitor the quality of these services and whether ethical standards are adhered to. It is important that professionals and agencies offering telepsychiatry services have professionals who are qualified and trained in this regard to some extent. This is just one of the few issues that concern mental health during the lockdown. ${ }^{5}$

\section{Relapse of Patients with Pre-Existing Psychiatric Diagnosis}

A burgeoning issue is one of patients with pre-existing psychiatric diagnoses. Procuring medications may be difficult in a lockdown due to short supply and there may relapses due to a lack of compliance with medication in many cases. Patients with schizophrenia may experience delusions and hallucinations and they may have new delusions that posit themselves around the themes of the current pandemic. Aggression and violent behavior may be exhibited by patients with schizophrenia. Patients with obsessive compulsive disorder may show a relapse of symptoms and the current status of sanitization and cleanliness may serve to exacerbate their obsessions and compulsions in the form repeated sanitizer use, repeated hand washing and rigorous cleanliness. Patients with panic disorder may have a resurgence of their panic symptoms and 
panic attacks may occur. Mood may go down and depressive symptoms may worsen while some patients may also develop suicidal thoughts and feelings. Withdrawal symptoms may be seen in patients with substance abuse to alcohol withdrawal and withdrawal from other drugs as they may not be available in the lockdown period. ${ }^{6-7}$

\section{Focus on Special Populations}

A major effect of the lockdown and COVID-19 would be on special populations that are vulnerable to be affected by mental health problems. Children and adolescents, geriatric populations, children with developmental disabilities, pregnant women and patients with medical illnesses that compound psychiatric illness need special mention. Many families may also undergo strife as they are staying together and there may be fights and squabbles between family members on a daily basis adding to the stress. Families must use this time to understand each other better and also end differences if any. ${ }^{8}$

\section{Mental Health of Healthcare Personnel}

All the the healthcare personnel and front line warriors looking after COVID patients and working in hospitals and isolation wards also need to have their mental health looked after. The stress of the work they are doing shall get to them and it will be difficult for them to deal with situations once cases escalate. Regular mental health care for these doctors, nurses and ancillary staff is very important for us to have a stable healthcare infrastructure to combat COVID. There is also a need for training these staff in communication skills and building their resilience for the tough times that they shall face ahead. ${ }^{9}$

\section{Mental Health of Sanitation Workers and Police Personnel}

Across the country, right from the onset of pandemic, police and sanitation workers are working continuously to help the government to control the spread of disease. They do service by staying away from families, with minimal facilities for food, water and other basic amenities. This group are vulnerable to severe stress due to prolonged work hours and pressure from the community and government to control the pandemic. Conducting regular counseling sessions, allowing them to ventilate their issues, conducting meditation, yoga camps will help them to cope up in long term.

\section{Patients with Severe Mental Illness and COVID}

Patients with severe mental illness may also be at an increased risk of contracting COVID infection. Homelessness and disorganization along with residences in slums may thwart social distancing protocols in these groups. Being illiterate and uneducated they will have little or no access to the COVID related educational material and may throw caution to the wind. Many patients with severe mental illness may delay diagnosis and coming to a hospital and may thus not receive mental health care. They may also delay seeking help due to lack of insight if having COVID and may also infect others around them. Even if treated they may not comply with medical care. Hence this is a very special group that needs attention during the pandemic. ${ }^{10}$

\section{Changes in Psychopharmacological Approaches}

Certain psychopharmacological tactics may need to be implemented in order to prevent withdrawal or side effect of drugs. In view of the medication shortage many stable patients with schizophrenia may be prescribed depot injectable antipsychotics to minimize the chances of withdrawal psychosis. Many patients on Benzodiazepines may face abrupt withdrawal with rebound anxiety and panic attacks. One must ensure that they get a prescription so that they have enough medicines to see them through the COVID period.

The unavailability of antidepressants, can cause withdrawal symptoms and rebound depression. One must shift patients on once a day longer antidepressants. There will be instances when patients of bipolar disorder on antiepileptics as a mood stabilizer may get a seizure due to abrupt withdrawal and shifting them to longer acting once a day preparation will be helpful while optimizing the dose. ${ }^{11}$

\section{Approach to ECT during COVID}

Many patients may have been in the middle of an electroconvulsive therapy (ECT) course when the pandemic started and must complete their course of ECT. It is imperative that adequate precautions be taken before ECT. We must screen patients before ECT procedure for respiratory symptoms, history of contact with COVID-19, monitor temperature and oxygen saturation well. We must actively liaison with the anesthesia team and ancillary staff and see that personal protection equipment is available for the staff. We must minimize the staff involved in ECT procedure and also plan that all patients needing ECT come to a single ECT room and on the same day. The patients receiving ECT must be adequately secluded from each other pre and post ECT to maintain physical distancing. One must give due attention to surface and equipment cleaning during and between cases (for example have a rigid protocol for anesthetic machine interface, bag, monitors, surfaces, door handles etc.). ${ }^{12}$

\section{Patients Visiting Psychiatric Clinics}

When patients visit the psychiatrist for a consultation, the clinic can be a potential source of infection for both people visiting the clinic and the staff working in it and this can be liability on the doctor running the clinic. Hence it may be best to screen patients for respiratory symptoms and provide them the option of teleconsultations if they have symptoms. In addition, a signed self-declaration for symptoms, travel history and informed consent can be obtained from patients and relatives.With little or no evidence base, treatment decisions need to be individualized to a given patient and psychiatrists have to use their clinical discretion based on the existing knowledge. Planning to prevent disruptions in the supply chain can help minimize the risk of withdrawals and relapse of symptoms. Psychiatrists need to liaise with the local public health authorities and develop plans to provide medications to patients at their homes. ${ }^{13}$ 


\section{Some other Factors}

There have been concerns over the role of mental disorders in corona virus transmission.

Patients with mental illness may not be aware of risks and may be uncooperative with regard to social distancing and personal protection. Once patients with mental illness are infected with severe acute respiratory syndrome corona virus 2 that shall cause COVID-19, more of them will be shunned away from mental health care. This will also happen due to the general discrimination associated with mental ill-health in health-care settings. The mental health disorder co-morbidities to COVID-19 will make the treatment more challenging and potentially less effective. COVID-19 has caused a parallel epidemic of fear, anxiety, and depression and this shall increase post the COVID epidemic as has been though of. People with mental health problems are affected by social media and the news brought on by the COVID-19 epidemic which causes a relapse of an already existing mental health condition because of high susceptibility to stress compared with the general population. ${ }^{14}$

\section{The Place of Psychiatry in COVID-19}

By seeing the rise of mental health issues, Government authorities have felt psychiatry as an essential field. Even Prime minister, Shri Narendra Modi had highlighted this in one of speeches during the corona crisis. District collectors and health administrators had initiated in formation of telecounselling centers in district hospitals to address psychological issues. Hospitals and many centers have allowed that their non-essential staff to stay home. In few areas and corporate sector, we may be considered nonessential as we are not trained in managing medical emergencies. But the question of essentiality and nonessential is a personal one that we have to answer. We cannot shy away as we are doctors first and psychiatrists later.

We have a practice that is spread out and flexible but in the midst of COVID-19, psychiatry and psychiatrists are essential and invaluable. We should be there for everyone who has fear and uncertainty related to the pandemic, even if they are not infected with COVID. These patients may come to casualty departments with fear and worry, may present with psychiatric symptoms or may be medical inpatients worried about their existing medical problems worsening and can even be psychiatric patients rattled by the current pandemic. ${ }^{15}$

Consultation-liaison psychiatry in shall play a vital role and will decide the best approach in this situation. Consultation-liaison psychiatrists work closely with the medical and surgical teams and also know about the worries that their colleagues undergo. Consultation-liaison psychiatrists usually traverse through the hospital and multiple specialties system and will be on the frontline in this pandemic. ${ }^{16}$

While we use telepsychiatry to manage our patients, it is vital that we realize we are as important on the frontline as we are on a video screen or telephone call.

\section{Acknowledgements}

Nil.

\section{Source of Funding \\ Nil}

\section{Conflict of Interest}

Nil

\section{References}

1. Sood S. Psychological effects of the Coronavirus disease-2019 pandemic. Res Hum Med Educ. 2020;7:23-6.

2. Qiu J, Shen B, Zhao M, Wang Z, Xie B, Xu Y. A nationwide survey of psychological distress among Chinese people in the COVID-19 epidemic: implications and policy recommendations. Gen Psychiatry. 2020;33(2):e100213.

3. Hawryluck L, Gold WL, Robinson S, Pogorski S, Galea S, Styra R. SARS control and psychological effects of quarantine, Toronto, Canada. Emerg Infect Dis. 2004;10(7):1206-12.

4. Brooks SK, Webster RK, Smith LE, Woodland L, Wessely S, Greenberg N, Rubin GJ. The psychological impact of quarantine and how to reduce it: rapid review of the evidence. Lancet. 2020;395(10227):912-20.

5. Whaibeh E, Mahmoud H, Naal H. Telemental Health in the Context of a Pandemic: the COVID-19 Experience. Curr Treat Options Psychiatry. 2020;2:1-5.

6. Chaturvedi SK. Covid-19, Coronavirus and Mental Health Rehabilitation at Times of Crisis. J Psychosoc Rehabil Ment Health. 2020;2:1.

7. Cowan HR. Is schizophrenia research relevant during the COVID-19 pandemic?. Schizophr Res. 2020.

8. Heymann DL, Shindo N. COVID-19: what is next for public health?. Lancet. 2020;395(10224):542-5.

9. Adams JG, Walls RM. Supporting the health care workforce during the COVID-19 global epidemic. JAMA. 2020 Mar 12 (published online).

10. Duan L, Zhu G. Psychological interventions for people affected by the COVID-19 epidemic. Lancet Psychiatry. 2020;7(4):300-2.

11. Xiang YT, Zhao YJ, Liu ZH, Li XH, Zhao N, Cheung T, Ng $\mathrm{CH}$. The COVID-19 outbreak and psychiatric hospitals in China: managing challenges through mental health service reform. Int J Biol Sci. 2020;16(10):1741-4.

12. Tor PC, Phu AAH, Koh DSH, Mok YM. ECT in a time of COVID-19. J ECT. 2020.

13. Liu S, Yang L, Zhang C, Xiang YT, Liu Z, Hu S, et al. Online mental health services in China during the COVID-19 outbreak. Lancet Psychiatry. 2020;7(4):e17-8.

14. Liebrenz M, Bhugra D, Buadze A, Schleifer R. Caring for persons in detention suffering with mental illness during the Covid-19 outbreak. Forensic Sci Int Mind Law. 2020;1:e100013.

15. Chen Q, Liang M, Li Y, Guo J, Fei D, Wang L, et al. Mental health care for medical staff in China during the COVID-19 outbreak. Lancet Psychiatry. 2020;7(4):e15-6.

16. Adams JG, Walls RM. Supporting the health care workforce during the COVID-19 global epidemic. JAMA. 2020.

How to cite this article: De Sousa A, Sai Krishna P. Psychosocial impact of the lockdown and COVID-19. Telangana J Psychiatry. 2020;6(1):4-6 